\title{
AUSLÄNDISCHE DIREKTINVESTITIONEN
}

Ausländische Direktinvestitionen (ADI) sind ein Schlüsselelement der internationalen wirtschaftlichen Integration. Durch ADI entstehen direkte, stabile und dauerhafte Beziehungen zwischen Volkswirtschaften. ADI fördern den Transfer von Technologie und Know-how zwischen den Ländern und ermöglichen es der Wirtschaft des Ziellands, ihre Produkte in größerem Umfang auf den internationalen Märkten abzusetzen. ADI stellen zudem eine zusätzliche Finanzierungsquelle für Investitionen dar und können in einem geeigneten politischen Umfeld ein wichtiges Instrument für die Entwicklung darstellen.

\section{Definition}

ADI sind grenzüberschreitende Investitionen, die von einem in einer Volkswirtschaft ansässigen Unternehmen mit der Absicht vorgenommen werden, eine dauerhafte Beteiligung an einem in einer anderen Volkswirtschaft ansässigen Unternehmen zu erwerben. Eine „dauerhafte Beteiligung“ impliziert das Bestehen einer langfristigen Geschäftsbeziehung zwischen dem Direktinvestor und dem Unternehmen sowie ein erhebliches Maß an Einflussnahme durch den Direktinvestor auf das Management des Unternehmens. Das grundlegende Kriterium ist ein Anteil von mindestens 10\% der Stimmrechte, durch den der Investor seinen Einfluss ausüben kann.

Der Bestand an Direktinvestitionen des Auslands bezieht sich auf sämtliche Unternehmensbeteiligungen, die von gebietsfremden Unternehmen zu einem gegebenen Zeitpunkt in der berichterstattenden Volkswirtschaft gehalten werden; beim Bestand an Direktinvestitionen im Ausland handelt es sich um die Unternehmensbeteiligungen, die von in der berichterstattenden Volkswirtschaft ansässigen Unternehmen im Ausland gehalten werden. Die entsprechenden $\mathrm{Zu}$ - und Abflüsse beziehen sich auf die Investitionen in einem bestimmten Zeitraum. Nettoabflüsse sind im Allgemeinen ein Anzeichen für Desinvestitionen oder eine Folge umfangreicher Darlehensrückzahlungen zwischen Unternehmen.

Der ADI-Index ist eine Messgröße für den Restriktionsgrad der für ausländische Direktinvestitionen geltenden Regeln eines Landes, bezogen auf vier Aspekte: Beschränkung ausländischer Kapitalbeteiligungen, Auswahl- oder Genehmigungsverfahren, Beschränkungen für die Beschäftigung von Ausländern in Schlüsselpositionen, Beschränkungen des operativen Geschäfts.

Die hier vorgestellten Indexwerte der OECD für den Restriktionsgrad der ADI-Regulierung zeigen, dass die tendenziell strengsten Beschränkungen für ausländische Direktinvestitionen im Dienstleistungssektor zu beobachten sind, gefolgt von den Primärsektoren. Das Verarbeitende Gewerbe ist nach wie vor der Wirtschaftssektor, der am stärksten geöffnet ist.

\section{Vergleichbarkeit}

Die Vergleichbarkeit der ADI-Statistiken hat sich in den letzten Jahren deutlich verbessert, doch bleiben Asymmetrien zwischen Direktinvestitionszuflüssen und -abflüssen bestehen.

\section{Überblick}

Die ADI-Aktivität verlangsamte sich 2011 nach einer kurzen Phase der Erholung im Jahr 2010. Die Direktinvestitionsabflüsse stiegen 2011 weltweit um 12\% auf 1558 Mrd. US-\$ an - gegenüber einer Zunahme um 24\% im Jahr 2010 - und lagen damit nach wie vor weit unter dem historischen Höchststand von 2007 (2 170 Mrd. US-\$). Auf Investoren aus OECD-Ländern entfielen rd. 83\% der weltweiten Direktinvestitionsabflüsse (1 $293 \mathrm{Mrd}$. US-\$), was einen Anstieg um $20 \%$ gegenüber 2010 darstellt. Die drei führenden ADI-Herkunftsländer waren die Vereinigten Staaten (419 Mrd. US-\$), Japan (114 Mrd. US-\$) und das Vereinigte Königreich (107 Mrd. US-\$), dessen Investitionen sich nach den seit 2009 beobachteten sehr niedrigen Niveaus in spektakulärer Weise erholten. Die Investoren aus der Europäischen Union (EU) insgesamt stellten 2011 mit 557 Mrd. US-\$ einen Anteil von $36 \%$ der weltweiten Direktinvestitionsabflüsse, womit ihr Anteil zwar seit 2009 unverändert blieb, aber weit unter dem Investitionsniveau von 2008 lag, als auf die EU 51\% der weltweiten Direktinvestitionsabflüsse entfielen.

In die OECD-Länder gingen nur 56\% (826 Mrd. US-\$) der weltweiten Direktinvestitionszuflüsse (gegenüber $87 \% \mathrm{im}$ Jahr 2000). Auf die Vereinigten Staaten entfielen allein $28 \%$ (234 Mrd. US-\$), was geringfügig mehr war als die kombinierten Direktinvestitionszuflüsse (225 Mrd. US-\$) nach Belgien (89 Mrd. US-\$), ins Vereinigte Königreich (54 Mrd. US-\$), nach Frankreich (41 Mrd. US-\$) und Deutschland (40 Mrd. US-\$). Die OECD-Investoren setzten die Diversifizierung der Zielländer ihrer Investitionen fort, wobei rd. 36\% ihrer Investitionen in Länder außerhalb des OECD-Raums gingen.

\section{Quelle}

- OECD (2012), OECD International Direct Investment Statistics (Datenbank).

- Foreign Direct Investment Statistics - OECD Data, Analysis and Forecasts, www.oecd.org/investment/statistics.

\section{Weitere Informationen}

\section{Analysen}

- OECD (2012), Annual Report on the OECD Guidelines for Multinational Enterprises, OECD Publishing.

- OECD (2012), OECD Investment Policy Reviews, OECD Publishing.

- OECD (2009), OECD Investment Policy Perspectives, OECD Publishing.

\section{Statistiken}

- OECD (2010), Measuring Globalisation: OECD Economic Globalisation Indicators, OECD Publishing.

\section{Zur Methodik}

- Kalinova, B., A. Palerm und S. Thomsen (2010), “OECD's FDI Restrictiveness Index: 2010 Update”, OECD Working Papers on International Investment, No. 2010/03.

- OECD (2008), OECD Benchmark Definition of Foreign Direct Investment, 4. Ausgabe, OECD Publishing.

- OECD (2005), Measuring Globalisation: OECD Handbook on Economic Globalisation Indicators, OECD Publishing.

\section{Websites}

- OECD International Investment, www.oecd.org/daf/investment. 
Bestand an Direktinvestitionen im Ausland und aus dem Ausland

Mio. US-Dollar

\begin{tabular}{|c|c|c|c|c|c|c|c|c|c|c|c|c|}
\hline & \multicolumn{6}{|c|}{ Direktinvestitionen im Ausland } & \multicolumn{6}{|c|}{ Direktinvestitionen des Auslands } \\
\hline & 1990 & 1995 & 2000 & 2009 & 2010 & 2011 & 1990 & 1995 & 2000 & 2009 & 2010 & 2011 \\
\hline Australien & 37491 & 60484 & 95978 & 343908 & 414298 & 382197 & 80333 & 111310 & 118858 & 428611 & 515981 & 554987 \\
\hline Belgien & 40636 & 80690 & 179773 & 891802 & 901924 & 970371 & 58388 & 112960 & 181650 & 946549 & 899252 & 998836 \\
\hline Chile & .. & .. & 11154 & 41339 & 49838 & 55602 & .. & .. & 45753 & 121395 & 139538 & 144729 \\
\hline Dänemark & .. & .. & 73117 & 213099 & 219900 & 242243 & .. & .. & 73585 & 154052 & 138747 & 145719 \\
\hline Deutschland & 130760 & 233107 & 486750 & 1346227 & 1405807 & 1406650 & 74067 & 104367 & 462564 & 944747 & 910381 & 902187 \\
\hline Estland & .. & .. & 256 & 6410 & 5698 & 4740 & .. & .. & 2611 & 16222 & 16474 & 16726 \\
\hline Finnland & 11227 & 14993 & 52109 & 129195 & 137042 & 138703 & 5132 & 8465 & 24272 & 84668 & 85588 & 82864 \\
\hline Frankreich & 110121 & 204430 & 445087 & 1492563 & 1536081 & 1581384 & 84931 & 191433 & 259773 & 985236 & 948442 & 953182 \\
\hline Griechenland & .. & & 5852 & 39457 & 42623 & 42936 & .. & .. & 14113 & 42101 & 35025 & 28079 \\
\hline Irland & .. & & 27925 & 289333 & 348733 & 313746 & .. & .. & 127088 & 247466 & 247094 & 251660 \\
\hline Island & 75 & 179 & 663 & 10180 & 11887 & 11302 & 147 & 129 & 497 & 8622 & 11784 & 12541 \\
\hline Israel & .. & 758 & 9091 & 57371 & 68973 & 71870 & 365 & 5741 & 22367 & 55797 & 60237 & 66554 \\
\hline Italien & 60195 & 106319 & 180274 & 486424 & 487610 & 512175 & 60009 & 65347 & 121169 & 364456 & 331960 & 332647 \\
\hline Japan & 201440 & 238452 & 278441 & 740965 & 831110 & 962790 & 9850 & 33508 & 50322 & 200151 & 214890 & 225785 \\
\hline Kanada & 84813 & 118106 & 237647 & 593580 & 616134 & 670417 & 112850 & 123182 & 212723 & 523247 & 561111 & 595002 \\
\hline Korea & .. & .. & .. & 120440 & 143160 & 160640 & .. & .. & .. & 121100 & 134230 & 135730 \\
\hline Luxemburg & .. & .. & .. & 192381 & 194257 & 194469 & .. & .. & .. & 150594 & 138172 & 142085 \\
\hline Mexiko & .. & .. & .. & 81216 & 104302 & 112088 & 22424 & 41130 & 97170 & 277898 & 330161 & 302305 \\
\hline Neuseeland & 3320 & 7676 & 6065 & 14737 & 16101 & 18979 & 8065 & 25728 & 28070 & 64801 & 67706 & 74299 \\
\hline Niederlande & 105085 & 172348 & 305458 & 956506 & 961526 & 978994 & 68699 & 115756 & 243730 & 660507 & 593101 & 582216 \\
\hline Norwegen & 10889 & 22521 & 22937 & 168871 & 185827 & .. & 12404 & 19836 & 25282 & 148315 & 154558 & . \\
\hline Österreich & 4747 & 11832 & 24820 & 163233 & 170363 & 195756 & 11098 & 21363 & 31165 & 172598 & 167958 & 164704 \\
\hline Polen & .. & 539 & 1018 & 29304 & 39029 & 50044 & 109 & 7843 & 34233 & 185182 & 201003 & 197538 \\
\hline Portugal & .. & .. & 19793 & 68477 & 66732 & 68048 & .. & 18973 & 32043 & 114718 & 111685 & 109028 \\
\hline Schweden & 50720 & 73143 & 123260 & 353376 & 368785 & 358886 & 12636 & 31089 & 93998 & 332108 & 349777 & 338484 \\
\hline Schweiz & 66087 & 142481 & 232176 & 826780 & 934126 & 991966 & 34245 & 57064 & 86810 & 492346 & 559333 & 583455 \\
\hline Slowak. Rep. & .. & 139 & 373 & 3152 & 3334 & 4209 & .. & 1297 & 4761 & 52541 & 50283 & 51290 \\
\hline Slowenien & .. & 727 & 870 & 9055 & 8175 & 7802 & .. & 2617 & 3278 & 15186 & 14466 & 15107 \\
\hline Spanien & 15652 & 31037 & 129192 & 625849 & 651314 & 640276 & 65916 & 110291 & 156347 & 632296 & 640799 & 634497 \\
\hline Tschech. Rep. & .. & 345 & 738 & 14805 & 14923 & 15470 & .. & 7350 & 21647 & 125829 & 128505 & 125245 \\
\hline Türkei & .. & .. & 3668 & 22250 & 22509 & 26398 & .. & .. & 19209 & 143724 & 185806 & 138752 \\
\hline Ungarn & .. & 278 & 1279 & 19244 & 19954 & 23756 & 569 & 11304 & 22856 & 99091 & 90783 & 84447 \\
\hline Ver. Königreich & 236118 & 330665 & 923366 & 1579715 & 1626819 & 1731065 & 233305 & 226626 & 463134 & 1104273 & 1162649 & 1198850 \\
\hline Ver. Staaten & 616655 & 885506 & 1531607 & 4029457 & 4306843 & 4681569 & 505346 & 680066 & 1421017 & 2398208 & 2597707 & 2908791 \\
\hline Euroraum & .. & .. & .. & 6713441 & 6934737 & 7068109 & .. & .. & .. & 5457875 & 5224897 & 5281510 \\
\hline EU27 & .. & .. & .. & 8928974 & 9230199 & 9495659 & .. & .. & .. & 7605056 & 7438191 & 7515827 \\
\hline OECD & 1786030 & 2736756 & 5410736 & 15960699 & 16915737 & .. & 1460888 & 1861984 & 4502095 & 12414636 & 12795187 & . \\
\hline Brasilien & ... & .. & ... & 164523 & 188637 & 202586 & .. & .. & .. & 400808 & 674764 & 669670 \\
\hline China & .. & .. & .. & 245800 & 310800 & 364200 & .. & .. & .. & 1314800 & 1476400 & 1804200 \\
\hline Indien & .. & .. & 2609 & 80943 & 96431 & 111267 & .. & .. & 20278 & 171437 & 204715 & 201743 \\
\hline Indonesien & .. & .. & 0 & 33 & 1731 & .. & .. & .. & .. & 108795 & 154158 & . \\
\hline Russ. Föderation & .. & 2420 & 20141 & 302542 & 366301 & 362101 & .. & 345 & 32204 & 378837 & 490560 & 457474 \\
\hline Südafrika & 15010 & 23301 & 32325 & 72583 & 89453 & .. & 9198 & 15014 & 43451 & 117434 & 153133 & . \\
\hline
\end{tabular}

StatLink ails $h t t p: / / d x . d o i . o r g / 10.1787 / 888932707420$

\section{Direktinvestitionsbestand}

In Prozent des BIP, 2011 oder letztes verfügbares Jahr

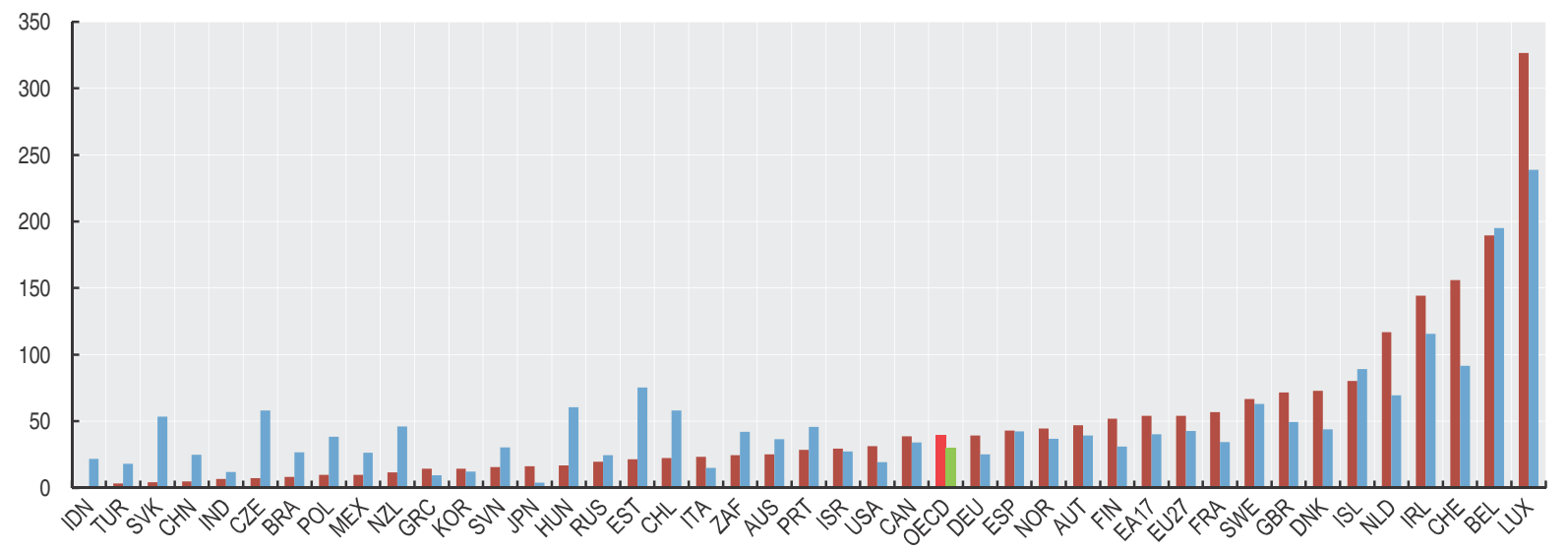


Direktinvestitionsabflüsse und Direktinvestitionszuflüsse

Mio. US-Dollar

\begin{tabular}{|c|c|c|c|c|c|c|c|c|c|c|c|c|}
\hline & \multicolumn{6}{|c|}{ Direktinvestitionsabflüsse } & \multicolumn{6}{|c|}{ Direktinvestitionszuflüsse } \\
\hline & 2006 & 2007 & 2008 & 2009 & 2010 & 2011 & 2006 & 2007 & 2008 & 2009 & 2010 & 2011 \\
\hline Australien & 25411 & 16855 & 33469 & 16693 & 27279 & 16197 & 31070 & 45530 & 47008 & 26554 & 33969 & 67471 \\
\hline Belgien & 50713 & 80141 & 220595 & 9207 & 55710 & 70772 & 58926 & 93448 & 193575 & 61748 & 81197 & 89222 \\
\hline Chile & 2171 & 2573 & 8041 & 8061 & 8743 & 8847 & 7298 & 12534 & 15150 & 12874 & 15095 & 14324 \\
\hline Dänemark & 8438 & 20624 & 13264 & 6320 & 3471 & 23677 & 2715 & 11815 & 1827 & 3942 & -7404 & 14806 \\
\hline Deutschland & 118767 & 170650 & 72617 & 75395 & 109328 & 54418 & 55657 & 80223 & 8093 & 24158 & 46863 & 40439 \\
\hline Estland & 1107 & 1746 & 1112 & 1549 & 142 & -1460 & 1797 & 2725 & 1729 & 1839 & 1600 & 257 \\
\hline Finnland & 4808 & 7202 & 9279 & 4917 & 10472 & 5333 & 7656 & 12455 & -1142 & 398 & 6733 & -47 \\
\hline Frankreich & 110734 & 164341 & 154747 & 107142 & 76878 & 90228 & 71888 & 96240 & 64060 & 24216 & 30634 & 40982 \\
\hline Griechenland & 4047 & 5247 & 2413 & 2055 & 978 & 1790 & 5358 & 2112 & 4490 & 2435 & 373 & 1825 \\
\hline Irland & 15332 & 21150 & 18912 & 26617 & 22350 & -4294 & -5545 & 24712 & -16421 & 25717 & 42807 & 11478 \\
\hline Island & 5555 & 10181 & -4206 & 2291 & -2357 & -29 & 3858 & 6822 & 917 & 86 & 246 & 1013 \\
\hline Israel & 15462 & 8604 & 7210 & 1695 & 9088 & 3080 & 15296 & 8798 & 10877 & 4438 & 5510 & 11374 \\
\hline Italien & 42089 & 90795 & 66870 & 21277 & 32657 & 47253 & 39259 & 40209 & -10814 & 20078 & 9179 & 29086 \\
\hline Japan & 50243 & 73545 & 127981 & 74698 & 56276 & 114300 & -6503 & 22548 & 24417 & 11938 & -1251 & -1758 \\
\hline Kanada & 44404 & 57719 & 79752 & 41728 & 38583 & 49566 & 59765 & 114642 & 57147 & 21438 & 23412 & 40929 \\
\hline Korea & 11175 & 19720 & 20251 & 17197 & 23278 & 20355 & 3586 & 1784 & 3311 & 2249 & 1094 & 4661 \\
\hline Luxemburg & 7183 & 73364 & 11737 & 7213 & 15124 & 11751 & 31803 & -28265 & 11195 & 22478 & 9211 & 17546 \\
\hline Mexiko & 5758 & 8256 & 1157 & 7019 & 13570 & 8946 & 20119 & 31492 & 27140 & 16119 & 20709 & 19554 \\
\hline Neuseeland & 182 & 3702 & -239 & -308 & 591 & 2798 & 4689 & 3440 & 4984 & -1293 & 636 & 3591 \\
\hline Niederlande & 71214 & 55618 & 68202 & 28182 & 55220 & 31896 & 13984 & 119406 & 4540 & 36044 & -8967 & 17145 \\
\hline Norwegen & 21321 & 13595 & 17298 & 30688 & 23085 & 20020 & 6413 & 5803 & 12254 & 14570 & 17518 & 3573 \\
\hline Österreich & 13678 & 39034 & 29395 & 10007 & 7732 & 30479 & 7936 & 31159 & 6845 & 9304 & 4265 & 14141 \\
\hline Polen & 8864 & 5410 & 4413 & 4701 & 5488 & 5870 & 19599 & 23582 & 14833 & 12936 & 8861 & 15165 \\
\hline Portugal & 7143 & 5494 & 2736 & 817 & -7494 & 12650 & 10914 & 3063 & 4656 & 2707 & 2646 & 10353 \\
\hline Schweden & 26613 & 38811 & 31298 & 25910 & 17969 & 26896 & 28908 & 27740 & 37120 & 10024 & -1348 & 12111 \\
\hline Schweiz & 75863 & 51036 & 45312 & 27845 & 64793 & 69545 & 43740 & 32446 & 15137 & 28696 & 20385 & -196 \\
\hline Slowak. Rep. & 512 & 600 & 529 & 432 & 328 & 491 & 4700 & 3583 & 4685 & -50 & 526 & 2145 \\
\hline Slowenien & 862 & 1865 & 1465 & 260 & -212 & 112 & 644 & 1515 & 1944 & -653 & 359 & 1000 \\
\hline Spanien & 104306 & 137078 & 74573 & 13072 & 38343 & 37290 & 30819 & 64277 & 76843 & 10406 & 40764 & 29504 \\
\hline Tschech. Rep. & 1469 & 1621 & 4322 & 950 & 1168 & 1155 & 5465 & 10446 & 6449 & 2929 & 6147 & 5417 \\
\hline Türkei & 924 & 2106 & 2549 & 1554 & 1464 & 2464 & 20185 & 22047 & 19504 & 8409 & 9038 & 15878 \\
\hline Ungarn & 3877 & 3622 & 2230 & 1987 & 1308 & 4541 & 7021 & 5447 & 6313 & 2051 & 2276 & 4709 \\
\hline Ver. Königreich & 82808 & 325473 & 182437 & 39325 & 39489 & 107076 & 156218 & 200068 & 88678 & 76375 & 50587 & 53944 \\
\hline Ver. Staaten & 244922 & 414039 & 329080 & 289450 & 327877 & 419332 & 243151 & 221166 & 310091 & 150443 & 205831 & 233988 \\
\hline Euroraum & 553411 & 855577 & 738200 & 308638 & 418323 & 386918 & 339469 & 550093 & 356525 & 245128 & 269999 & 306340 \\
\hline EU27 & 686543 & 1252669 & 977776 & 387804 & 487525 & 556613 & 582075 & 855856 & 538685 & 361775 & 334791 & 419796 \\
\hline OECD & 1187954 & 1931815 & 1640800 & 905945 & 1078720 & 1293347 & 1008391 & 1355010 & 1057433 & 645847 & 679498 & 825630 \\
\hline Brasilien & 28202 & 7067 & 20457 & -10084 & 11589 & -1029 & 18822 & 34585 & 45058 & 25949 & 48506 & 66661 \\
\hline China & 21200 & 17000 & 53500 & 43900 & 60100 & 43000 & 124100 & 160100 & 175100 & 114200 & 185000 & 228600 \\
\hline Indien & 14344 & 17281 & 19257 & 15928 & 14789 & 12416 & 20336 & 25483 & 43407 & 35597 & 25882 & 34247 \\
\hline Indonesien & 2726 & 4675 & 5900 & 2249 & 2664 & 7771 & 4914 & 6929 & 9318 & 4878 & 13771 & 18906 \\
\hline Russ. Föderation & 23151 & 45916 & 55594 & 43666 & 52523 & 67283 & 29701 & 55073 & 75002 & 36500 & 43288 & 52878 \\
\hline Südafrika & 6063 & 2966 & -3134 & 1151 & -76 & -635 & -527 & 5695 & 9007 & 5696 & 1228 & 5807 \\
\hline
\end{tabular}

\section{Direktinvestitionsströme}

\section{Mrd. US-Dollar, 2011}

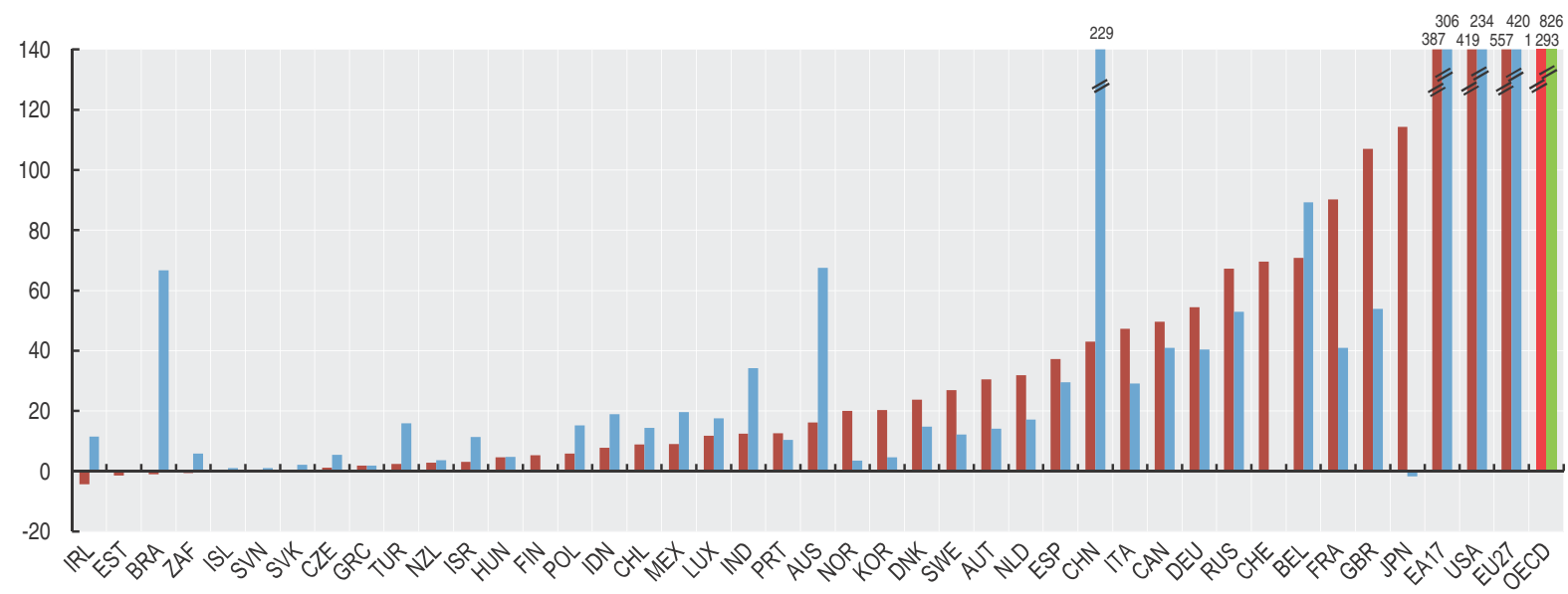


Index für den Restriktionsgrad der ADI-Regulierung

2011

\begin{tabular}{|c|c|c|c|c|c|c|c|c|c|c|}
\hline & ADI-Index insg. & Primärsektor & $\begin{array}{l}\text { Verarbeitendes } \\
\text { Gewerbe }\end{array}$ & Stromsektor & Vertrieb & Verkehr & Medien & Kommunikation & $\begin{array}{c}\text { Finanz- } \\
\text { dienstleistungen }\end{array}$ & $\begin{array}{l}\text { Unternehmens- } \\
\text { dienstleistungen }\end{array}$ \\
\hline Australien & 0.128 & 0.078 & 0.075 & 0.075 & 0.075 & 0.267 & 0.200 & 0.400 & 0.133 & 0.078 \\
\hline Belgien & 0.040 & 0.035 & 0.023 & 0.023 & 0.023 & 0.114 & 0.023 & 0.023 & 0.024 & 0.248 \\
\hline Chile & 0.068 & 0.150 & 0.000 & 0.000 & 0.000 & 0.413 & 0.413 & 0.000 & 0.017 & 0.013 \\
\hline Dänemark & 0.072 & 0.056 & 0.000 & 0.000 & 0.000 & 0.083 & 0.000 & 0.000 & 0.002 & 0.363 \\
\hline Deutschland & 0.023 & 0.069 & 0.000 & 0.000 & 0.000 & 0.200 & 0.025 & 0.000 & 0.005 & 0.000 \\
\hline Estland & 0.022 & 0.000 & 0.000 & 0.000 & 0.000 & 0.150 & 0.000 & 0.000 & 0.002 & 0.000 \\
\hline Finnland & 0.019 & 0.015 & 0.009 & 0.084 & 0.009 & 0.092 & 0.009 & 0.009 & 0.011 & 0.046 \\
\hline Frankreich & 0.045 & 0.155 & 0.000 & 0.000 & 0.000 & 0.150 & 0.048 & 0.000 & 0.054 & 0.003 \\
\hline Griechenland & 0.039 & 0.079 & 0.000 & 0.000 & 0.000 & 0.150 & 0.113 & 0.000 & 0.020 & 0.056 \\
\hline Irland & 0.043 & 0.135 & 0.000 & 0.000 & 0.000 & 0.125 & 0.000 & 0.000 & 0.009 & 0.000 \\
\hline Island & 0.231 & 0.463 & 0.112 & 1.000 & 0.112 & 0.295 & 0.112 & 0.112 & 0.119 & 0.112 \\
\hline Israel & 0.118 & 0.060 & 0.020 & 0.770 & 0.020 & 0.403 & 0.264 & 0.396 & 0.037 & 0.020 \\
\hline Italien & 0.050 & 0.130 & 0.000 & 0.000 & 0.000 & 0.150 & 0.363 & 0.000 & 0.018 & 0.000 \\
\hline Japan & 0.265 & 1.000 & 0.077 & 0.000 & 0.000 & 0.667 & 0.200 & 0.480 & 0.000 & 0.000 \\
\hline Kanada & 0.166 & 0.188 & 0.100 & 0.100 & 0.100 & 0.267 & 0.700 & 0.625 & 0.067 & 0.100 \\
\hline Korea & 0.143 & 0.250 & 0.000 & 0.417 & 0.000 & 0.508 & 0.563 & 0.500 & 0.050 & 0.000 \\
\hline Luxemburg & 0.004 & 0.000 & 0.000 & 0.000 & 0.000 & 0.075 & 0.000 & 0.000 & 0.002 & 0.000 \\
\hline Mexiko & 0.225 & 0.394 & 0.103 & 0.100 & 0.175 & 0.528 & 0.663 & 0.350 & 0.133 & 0.100 \\
\hline Neuseeland & 0.240 & 0.325 & 0.200 & 0.200 & 0.200 & 0.283 & 0.200 & 0.400 & 0.233 & 0.200 \\
\hline Niederlande & 0.015 & 0.062 & 0.000 & 0.000 & 0.000 & 0.083 & 0.000 & 0.000 & 0.002 & 0.000 \\
\hline Norwegen & 0.080 & 0.156 & 0.000 & 0.000 & 0.000 & 0.350 & 0.125 & 0.000 & 0.033 & 0.313 \\
\hline Österreich & 0.106 & 0.150 & 0.000 & 1.000 & 0.000 & 0.182 & 0.000 & 0.000 & 0.002 & 0.322 \\
\hline Polen & 0.072 & 0.050 & 0.000 & 0.000 & 0.000 & 0.092 & 0.298 & 0.075 & 0.003 & 0.000 \\
\hline Portugal & 0.007 & 0.006 & 0.000 & 0.000 & 0.000 & 0.083 & 0.000 & 0.000 & 0.017 & 0.000 \\
\hline Schweden & 0.059 & 0.138 & 0.000 & 0.000 & 0.000 & 0.292 & 0.200 & 0.200 & 0.002 & 0.051 \\
\hline Schweiz & 0.083 & 0.000 & 0.000 & 0.500 & 0.000 & 0.250 & 0.467 & 0.000 & 0.067 & 0.000 \\
\hline Slowak. Rep. & 0.049 & 0.000 & 0.000 & 0.000 & 0.000 & 0.075 & 0.000 & 0.000 & 0.002 & 0.000 \\
\hline Slowenien & 0.007 & 0.000 & 0.000 & 0.000 & 0.000 & 0.150 & 0.000 & 0.000 & 0.002 & 0.000 \\
\hline Spanien & 0.021 & 0.011 & 0.000 & 0.000 & 0.000 & 0.075 & 0.225 & 0.000 & 0.002 & 0.113 \\
\hline Tschech. Rep. & 0.055 & 0.025 & 0.000 & 0.000 & 0.000 & 0.075 & 0.000 & 0.000 & 0.010 & 0.000 \\
\hline Türkei & 0.077 & 0.013 & 0.000 & 0.000 & 0.000 & 0.383 & 0.125 & 0.000 & 0.000 & 0.125 \\
\hline Ungarn & 0.049 & 0.000 & 0.000 & 0.000 & 0.000 & 0.167 & 0.000 & 0.000 & 0.005 & 0.000 \\
\hline Ver. Königreich & 0.061 & 0.160 & 0.023 & 0.023 & 0.023 & 0.114 & 0.248 & 0.023 & 0.024 & 0.023 \\
\hline Ver. Staaten & 0.089 & 0.181 & 0.000 & 0.197 & 0.000 & 0.550 & 0.250 & 0.110 & 0.042 & 0.000 \\
\hline EU27 & & & & & & .. & .. & & & \\
\hline OECD & 0.081 & 0.133 & 0.022 & 0.132 & 0.022 & 0.231 & 0.171 & 0.109 & 0.034 & 0.067 \\
\hline Brasilien & 0.086 & 0.188 & 0.025 & 0.025 & 0.025 & 0.275 & 0.488 & 0.025 & 0.025 & 0.025 \\
\hline China & 0.409 & 0.454 & 0.193 & 0.463 & 0.238 & 0.633 & 1.000 & 0.750 & 0.525 & 0.350 \\
\hline Indien & 0.300 & 0.405 & 0.053 & 0.050 & 0.431 & 0.263 & 0.463 & 0.425 & 0.313 & 0.563 \\
\hline Indonesien & 0.311 & 0.286 & 0.070 & 0.110 & 0.435 & 0.423 & 1.000 & 0.410 & 0.206 & 0.579 \\
\hline Russ. Föderation & 0.180 & 0.150 & 0.092 & 0.030 & 0.050 & 0.350 & 0.117 & 0.100 & 0.515 & 0.175 \\
\hline Südafrika & 0.054 & 0.010 & 0.010 & 0.010 & 0.010 & 0.193 & 0.298 & 0.010 & 0.043 & 0.260 \\
\hline
\end{tabular}

Index für den Restriktionsgrad der ADI-Regulierung

2011

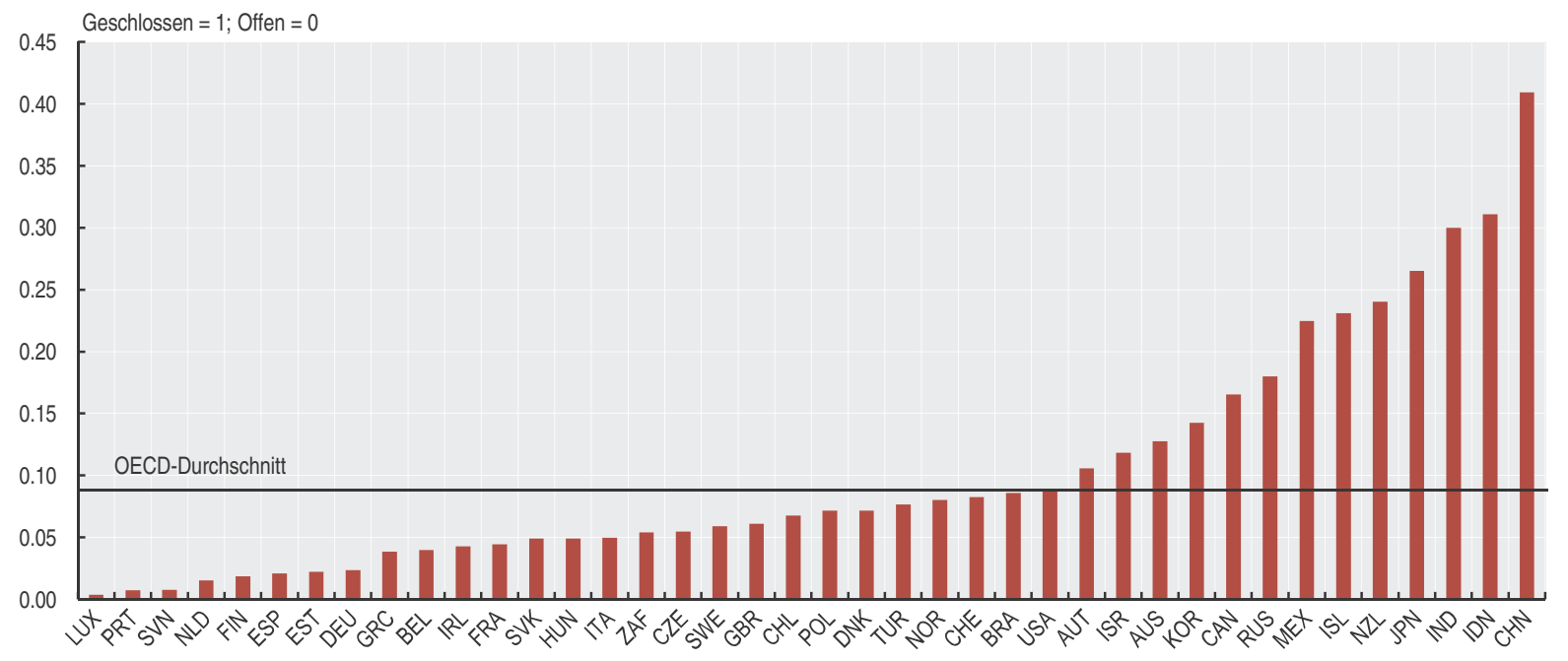

StatLink 霆及 $h$ ttp://dx.doi.org/10.1787/888932707515 


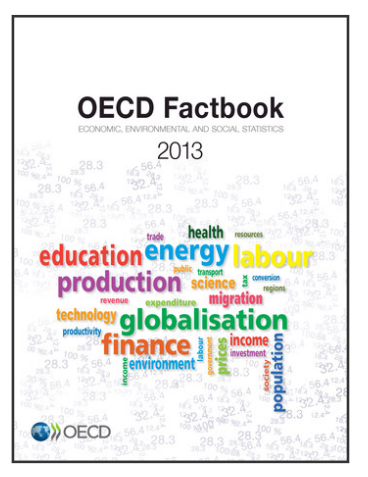

\section{From: \\ OECD Factbook 2013 \\ Economic, Environmental and Social Statistics}

Access the complete publication at:

https://doi.org/10.1787/factbook-2013-en

\section{Please cite this chapter as:}

OECD (2013), "Ausländische Direktinvestitionen”, in OECD Factbook 2013: Economic, Environmental and Social Statistics, OECD Publishing, Paris.

DOI: https://doi.org/10.1787/factbook-2013-34-de

Das vorliegende Dokument wird unter der Verantwortung des Generalsekretärs der OECD veröffentlicht. Die darin zum Ausdruck gebrachten Meinungen und Argumente spiegeln nicht zwangsläufig die offizielle Einstellung der OECDMitgliedstaaten wider.

This document and any map included herein are without prejudice to the status of or sovereignty over any territory, to the delimitation of international frontiers and boundaries and to the name of any territory, city or area.

You can copy, download or print OECD content for your own use, and you can include excerpts from OECD publications, databases and multimedia products in your own documents, presentations, blogs, websites and teaching materials, provided that suitable acknowledgment of OECD as source and copyright owner is given. All requests for public or commercial use and translation rights should be submitted to rights@oecd.org. Requests for permission to photocopy portions of this material for public or commercial use shall be addressed directly to the Copyright Clearance Center (CCC) at info@copyright.com or the Centre français d'exploitation du droit de copie (CFC) at contact@cfcopies.com. 